สี

Low Power RF Beam Control Electronics for the LEB

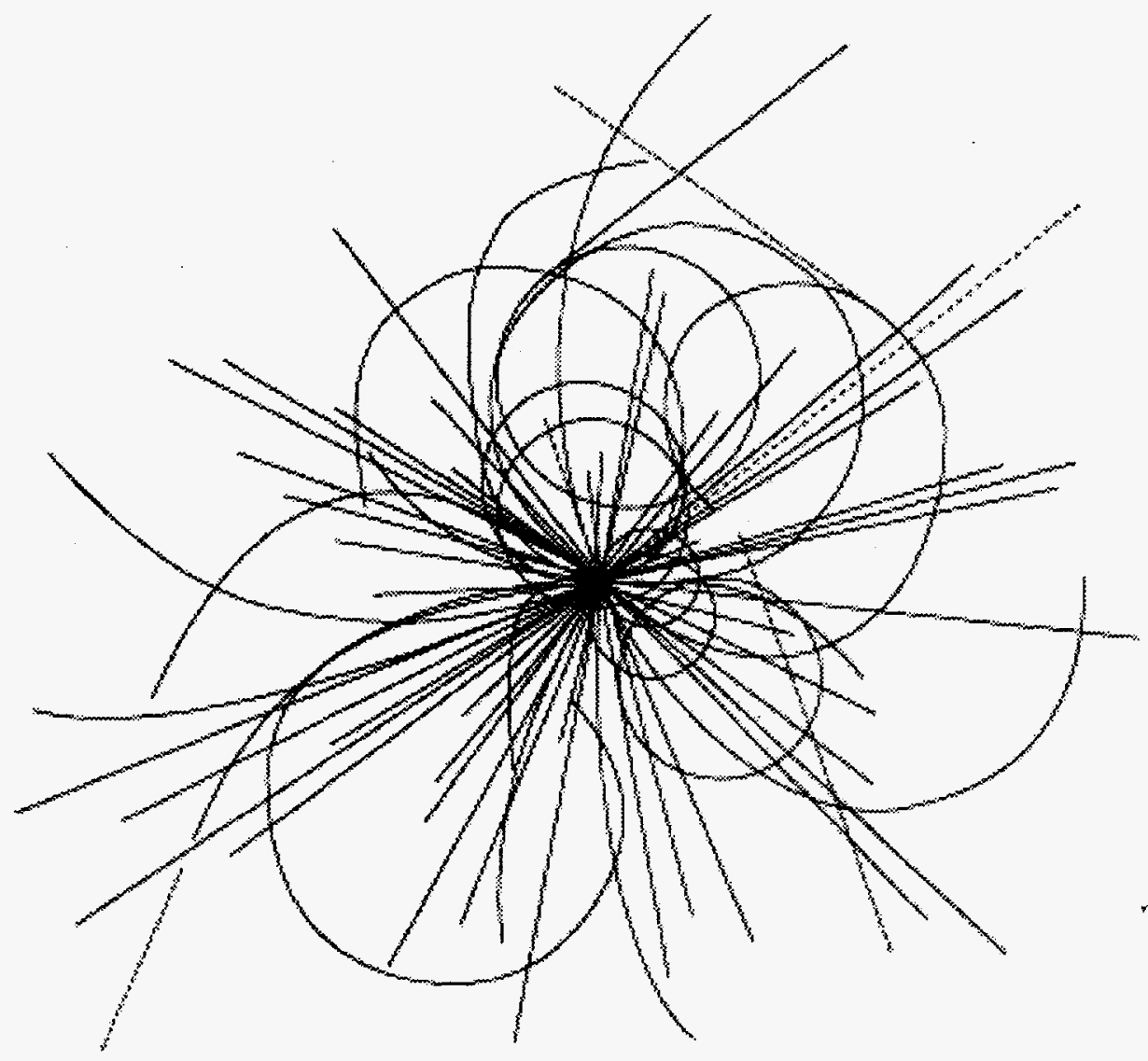

\section{Superconducting Super Collider Laboratory}

CONF-93053)--119 SSCL-Preprint-221

May 1993

Distribution Category: 400

L. K. Mestha

J. Mangino

V. Brouk

T. Uher

R. C. Webber
APPRONEO FOR RELEASE OR

PUBLOATION - O.R.PATEMT GROUP BY. Qf. ...... OATE. 4 . 35 


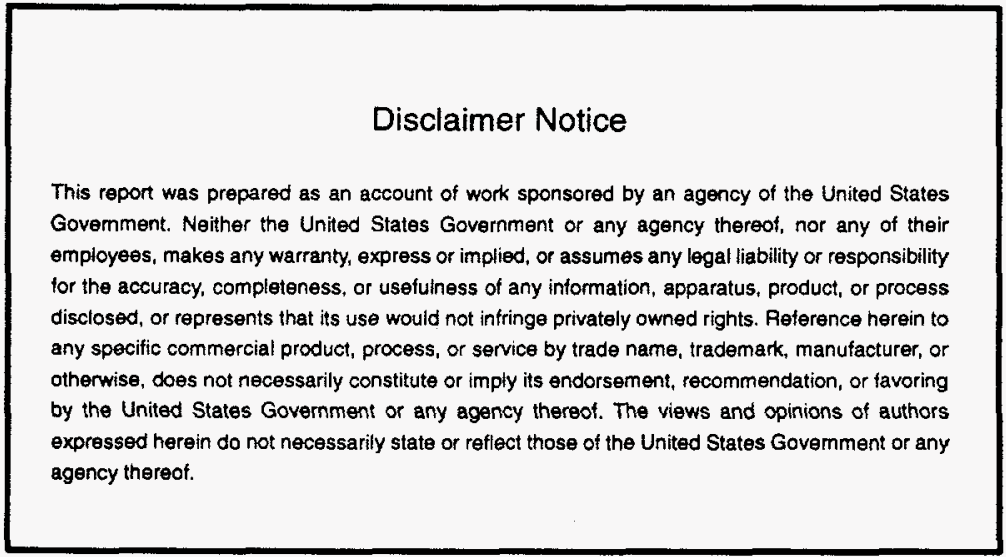

Superconducting Super Collider Laboratory is an equal opportunity employer. 


\section{DISCLAIMER}

Portions of this document may be illegible in electronic image products. Images are produced from the best available original document. 


\section{Low Power RF Beam Control} Electronics for the LEB*

L. K. Mestha, J. Mangino, V. Brouk, T. Uher, and R. C. Webber

Superconducting Super Collider Laboratory ${ }^{\dagger}$

2550 Beckleymeade Ave.

Dallas, TX 75237

May 1993

*Presented at the Fifth Annual International Symposium on the Super Collider, May 6-8, 1993 San Francisco, CA. tOperated by the Universities Research Association, Inc., for the U.S. Department of Energy under Contract No. DE-AC35-89ER40486. 


\title{
LOW POWER RF BEAM CONTROL ELECTRONICS FOR THE LEB
}

\author{
L.K. Mestha, J. Mangino, V. Brouk, T. Uher and R. C. Webber \\ Superconducting Super Collider Laboratory* \\ 2550 Beckleymeade Ave., Dallas, TX 75237
}

\begin{abstract}
Beam Control Electronics for the Low Energy Booster (LEB) should provide a fine reference phase and frequency for the High Power RF System. Comections applied on the frequency of the if signal will reduce dipole synchrotron oscillations due to power supply regulation errors, errors in frequency source or errors in the cavity voltage. It will allow programmed beam radial position control throughout the LEB acceleration cycle. Furthermore the if signal provides necessary corrections during adiabatic capture of the beam as injected into the LEB by the Linac and will guarantee LEB if phase synchronism with the Medium Energy Booster (MEB) if at a programmed time in the LEB cycle between a unique LEB bucket and a unique MEB bucket. We show in this paper a manured design and possible interfaces with other subsystems of the LEB such as the beam instrumentation, High Power RF Stations, global accelerator controls and the precision timing system. The outline of various components of the beam control system is also presented followed by some test results.
\end{abstract}

\section{INTRODUCTION}

Some early thoughts on the overall design and development of the Low Power RF control electronics were presented in earlier conferences on the Super Collider. ${ }^{1,2}$ Particularly in Reference 1 the new beam transfer synchronization was discussed without any experimental results. Upon thorough investigation of the machine requirements and due to considerable progress in the design of other LEB subsystems we were able to design a more matured system. Some of the redundant hardware was taken out from the design shown in Reference 2 . Due to the clear picture of the link with the SSC precision timing system we are now able to show for the first time the beam synchronization system working together with the essential components of the timing system. Among the test results shown at the end of this paper, some were done with beam on Fermilab booster.

\section{ESSENTIAL PARTS OF BEAM CONTROL LOOP HARDWARE}

Generally, loops associated with the beam control are (1) Beam Phase Loop, (2) Radial Loop, and (3) Synchronization Loop. We have shown schematically in Figure 1 a top level block diagram of digital implementation. The Direct Digital Synthesizer (DDS) uses 1-Ghz clock to produce the frequency between $47 \mathrm{Mhz}$ to $60 \mathrm{Mhz}$ and a strobe signal to latch the data. Since the Synthesizer needs data in the form of a 32-bit binary word the hardware is digital. Digital Signal Processor (DSP) \#1 is used to produce the basic frequency ramp.

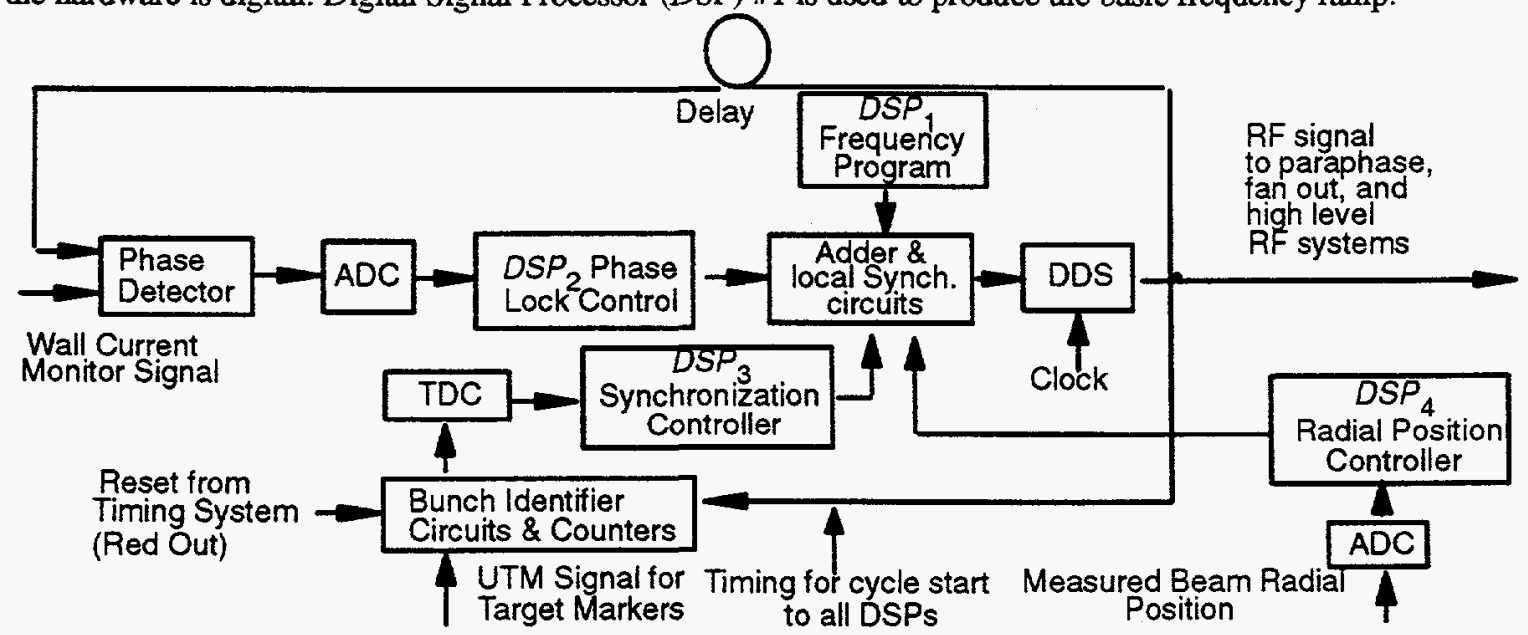

Figure 1. Schematic diagram of the Synchronization and Beam Control System.

\section{Beam Phase Loop}

The beam phase loop has a Phase Detector, a 12-bit ADC, a Digital Signal Processor \#2 and additional hardware with external adder circuits. The beam signal is compared with the Synthesizer rf signal through a delay cable in a beam phase detector. The beam phase is then digitized using the 12-bit ADC and the data is read into the DSP. The beam phase data is multiplied inside the Signal Processor by a time varying gain (if needed). Additional

\footnotetext{
*Operated by the Universities Research Association, Inc., for the U.S. Department of Energy under Contract No. DE-AC35-89ER40486.
} 
filters or non-linear controllers (if found useful) are used in the Processor on real time. The ADC is sampled at DDS strobe signal period. With the hardware we have used in the beam phase loop, a sampling period of less than $3 \mu s$ has been achieved in Laboratory tests. For the total phase lag to be less than 45 degrees introduced in the beam phase loop at twice the maximum synchrotron frequency (the power amplifier to cavity, beam, the sensor such as a wall current monitor, cables, phase detector and digital processing), a stringent requirement is placed on the sampling period. In case the delay turns out to be larger than expected then the plan is to replace the phase loop DSP with analog processing and do the analog to digital conversion afterwards.

\section{Synchronization Loop}

The synchronization loop shown in Figure 1 has been designed to phase lock the LEB if signal to the MEB if signal with the MEB running at its injection frequency. LEB revolution markers and the MEB target markers are used for locking the frequencies. The target markers are derived from the MEB rf signal by dividing its frequency by the harmonic number (792) and displacing the marker train appropriately for multiple transfer. This is done in a timing module and is discussed later in this document. The revolution markers for the LEB $f$ signal are derived by dividing the LEB if signal by the harmonic number (114) after identifying a particular bucket to track. This is done in 'bunch identifying circuits' of Figure 1. Two revolution markers are used as 'Ref' and 'Hit' for the Time to Digital Converter (IDC). A 20-ps TDC is used in the synchronization loop. Marking the bucket from the LEB rf signal is shown in the timing diagram of Figure 2. The 'reset' pulse in this figure is generated from MCLK (MEB beam synchronous clock - same as MEB rf signal, but with coded messages sent over the timing system) and is shown in the Interface section below. The 'reset' pulse indicates the arrival of the first target marker. After the arrival of the first target marker, count the pulses from the LEB if signal. After a known number of count (say 40 or any bucket we want to identify) the $\mathrm{ff}$ signal is divided by the harmonic number, 114, to generate the revolution marker. This means we have identified a 40th bucket and are tracking at each revolution in the MEB. The TDC will output the time interval between two markers. The Signal Processor \#3 will read the TDC data each time a new data appears and the new data appears each time the target marker appears. In the synchronization DSP, the time interval is processed and then compared to the 'trip-plan' values. The trip-plan values indicate the position of the LEB reference bunch in the ring when the MEB target marker arrives. These values can be calculated (theoretical) or measured. The data representing the error between the urip-plan values and the processed TDC values are then multiplied by a constant to produce the frequency shift on the adder interface circuits at the input end of the DDS. In this way, the hardware provides the ability to phase lock the MEB rf signal to the LEB rf signal. Time for closing the synchronization loop can be done at a predetermined count of the MEB target marker. In this way when the synchronization loop is closed the LEB if signals are phase matched to the trip-plan data.

Since the trip-plan values are known in advance, we can select a particular count on the MEB target markers after reaching the required momentum to fire the kickers. Say for 3770 counts of the target markers we would have reached the target momentum (indication of the momentum match with the MEB can come from a gauss clock looking at the LEB main magnetic field) and are within the momentum tolerance of the MEB. Then, the LEB beam can be extracted any time. However, if there is a requirement on firing the kickers to phase match to the gap in the LEB, then there is no change required for the synchronization loop because the reference bucket created in the bunch identifier circuits can be made to phase match with the gap.

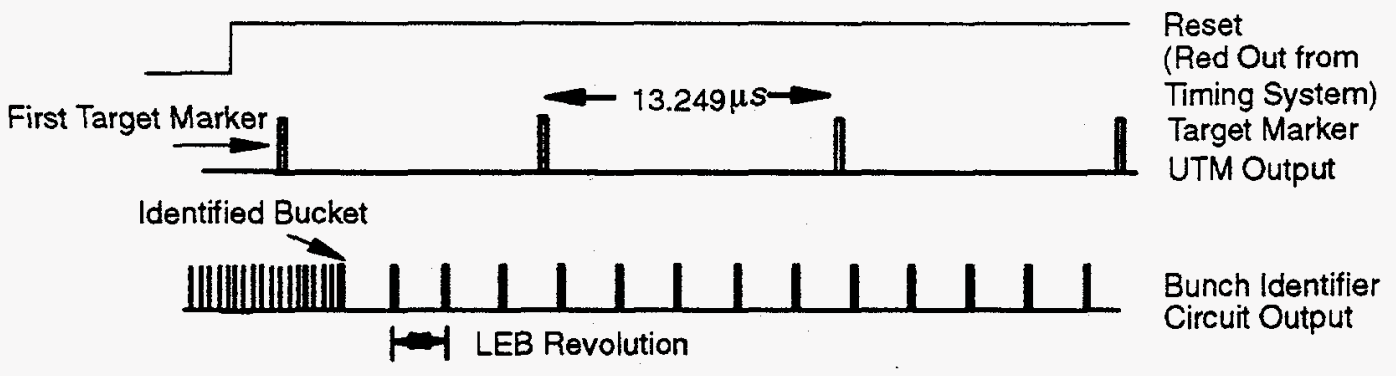

Figure 2. Basic timing diagram of the synchronization loop.

\section{Radial Loop}

With increased understanding of the feedback loops the radial loop is planned to configure differently from that of the Fermilab booster. The radial position signal is digitized using a 12-bit ADC and is read into DSP \#4. The radial position values are compared with the desired reference data inside the signal processor. Resulting data is multiplied by the time varying gain and then converted to frequency shift and applied to the adder circuits. In this way the radial loop is producing additional frequency modulation on the DDS signal. 


\section{INTERFACES WITH OTHER SUBSYSTEMS}

\section{Interface with Beam Instrumentation}

Beam instrumentation interface is needed to acquire on real time the longitudinal and radial position information of the beam. Unprocessed resistive wall current monitor is used to detect the longitudinal position. The wall current monitor is a device measuring the beam current almost virtually with a flat amplitude response. It is a 'current transformer' responding to currents in the vacuum chamber which were created largely due to the beam travelling down the pipe. The shape and the size of the wall current monitor signal depends on the particle distribution in the beam tube and the number of particles. ${ }^{3}$ For the LEB, a signal dynamic range of $48 \mathrm{~dB}$ is expected for operations with particles per bunch of $2.0 \times 10^{8}$ in commissioning mode to $5.0 \times 10^{10}$ in test beam mode. The signal from the wall current monitor is connected to the beam phase detector as shown in Figure 1. For operations with such a wide dynamic range the signal processing electronics in the beam phase detector needs additional circuits which could be made switchable depending on the beam intensity.

A VXI card (block diagram is shown in Figure 3) which gives the processed 'sum' and 'difference' signals of the horizontal beam position monitor electrodes will be used to measure the radial position. This card will be located in the LEB RF building (Building S2) along with the Synchronization and Beam Control electronics. As shown in Figure 3 the output of two horizontal electrodes placed at the opposite sides of the vacuum chamber (Electrodes A \& B) of the Beam Position Monitor (BPM) are used to measure the radial position. The beam's radio frequency component is selected and logarithmically demodulated. The difference of the output of the logarithmic amplifiers of the signal processing circuits produces a voltage proportional to the beam position. A prototype board was developed and tested which contained a special circuit topology and modern analog circuit components to obtain a low noise, high bandwidth and wide dynamic range in position measurement. ${ }^{4}$ Particularly for the radial loop a bandwidth of few kilohertz is found sufficient. The BPM closest to arc A1 is designated for the radial feedback which has a highest dispersion of 2.7 in the ring.

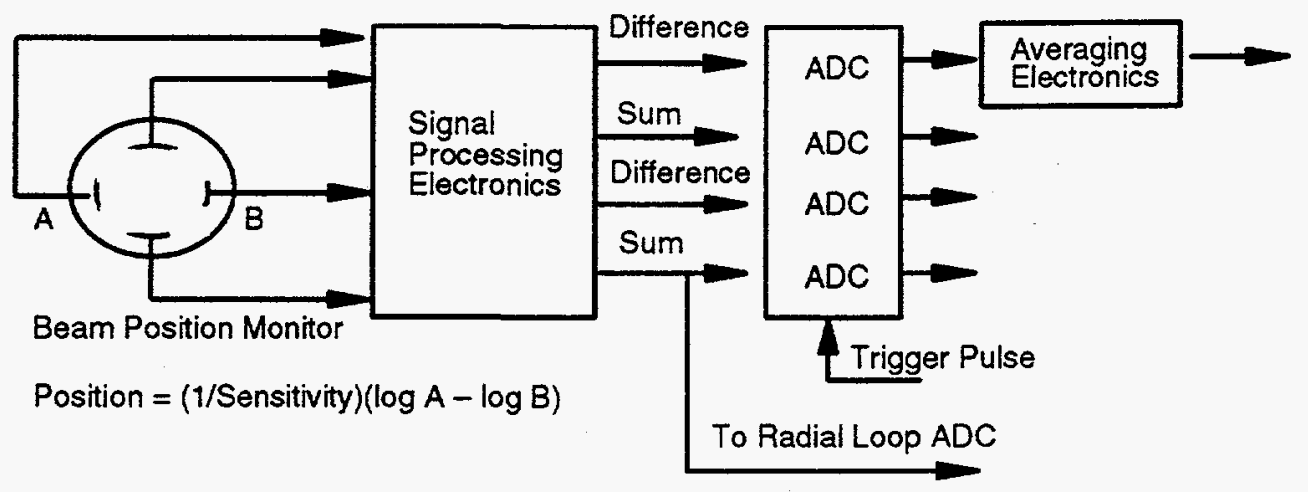

Figure 3. Radial position detector and processing circuits.

\section{Interface with the Timing System}

The LEB Low Power RF and other electronics for the if systems are located in LEB RF building. Similarly the MEB RF electronics are located in the MEB RF building. For phase matching the MEB bucket with the LEB bucket the MEB if signal is brought over a special timing cable from the MEB RF building to the LEB RF building. This signal is called MCLK - the MEB beam synchronous clocks of the timing system. The electronics required for firing the kicker system are placed in the LEB extraction building. The codes on global precision timing system carry information required to fire the LEB and MEB kickers. In Figure 4 we show a typical top level timing diagram of generating the target markers from the codes. Codes such as 'black' appear on the 'MCLK' timing signal at the end of every MEB revolution marker. This code is associated with a delay of say ' $d_{1}$ ' in the target markers. Since the target markers happen to be the destination bucket for the LEB reference bunch, the delays ' $\mathrm{d}_{1}$ ', ' $\mathrm{d}_{2}$ ', ' $\mathrm{d}_{3}$ ', etc., will be needed to phase shift them for multiple transfer from a common reference. Each delay is associated with a particular color code on the MCLK timing signal. The black code will get changed to red code when LEB reset is detected which is associated with a state change on the signal line 'red out' to high. At the same time the 'black out' signal goes low to indicate the elapse of black code. The encoder in the timing system will detect the red code and delay the target markers by ' $d_{2}$ ' as indicated by the 'Target Markers' signal. The first target pulse after the 'red out' signal goes active high. This will be used to identify the first target marker for beam transfer synchronization. Since the synchronization is guaranteed after a definite number of MEB if counts after the first target marker, all the power supplies associated with the kicker charging and firing system can be timed out of this pulse to extract the beam from the LEB.

The 'LEB Reset' is coded on GCLK timing signal. The reset appears at $10 \mathrm{~Hz}$, which is derived from the main magnet field or current measurement system to time the injection of the Linac beam before the field minimum on the LEB magnet system. The 'LEB Reset' signal is used to ramp the frequency in the DDS and various other cavity tuning functions in the High Power RF System. This signal is asynchronous to the target markers. Hence an uncertainty of one MEB turn with respect to the 'trip-plan' is expected while identifying the target markers after the LEB reset has gone active high. Since all the kicker system including the bunch identifier circuits in the synchronization system ( Figure 1) will see the same first target marker, there will be no mismatch of the target marker counts. However, the synchronization loop will cure any phase errors to maintain the required phase relationship with the target markers by maneuvering the radial orbit. 


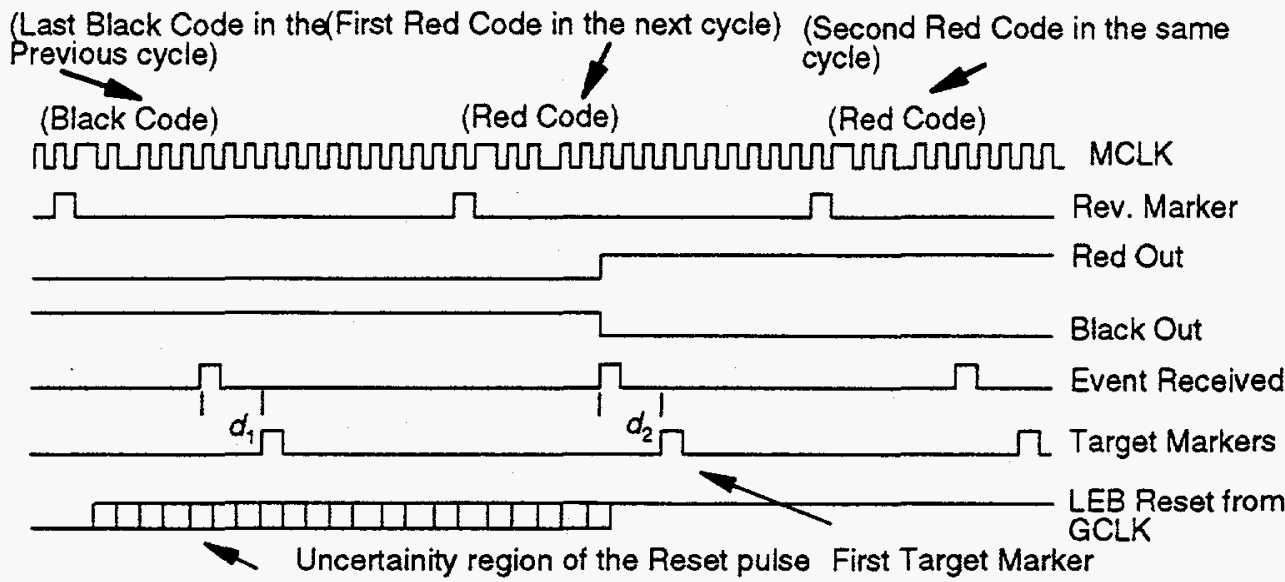

Figure 4. Top level timing diagram of the target marker generator.

\section{Interface with High Power RF System}

Analog Interface. The DDS signal is fanned out to all the LEB cavities through a delay unit or a phase shifter as shown in Figure 5. The programmable phase shifters are used to generate required counterphasing between the selected pair of cavities. At this stage it is unclear as to how many pairs of cavities are involved during counterphasing. Also the feedback loops local to the if cavities are expected to maintain the phase constant between the if signal and the gap voltage during acceleration.

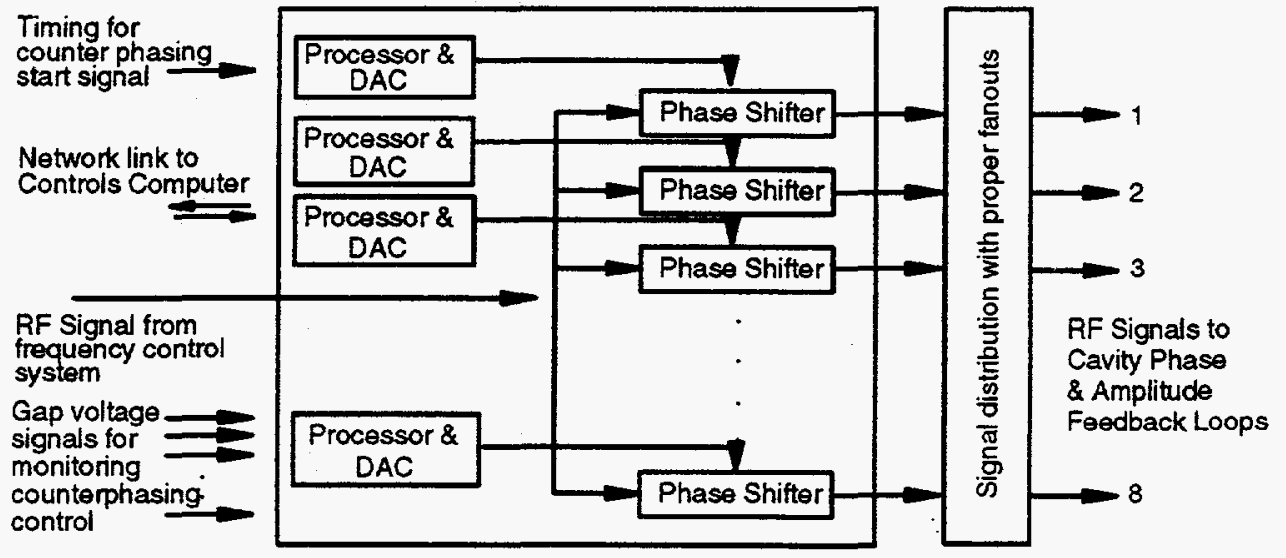

Figure 5. Schematic diagram of the counter phase control units.

Digital/Computer Interface. A schematic layout of the planned data and control flow is compiled in Figure 6. The synchronization and beam control hardware is resident in if group control area. In addition to generating an accurate if signal with appropriate counterphasing, the voltage profiles, cavity tuner function profiles and power amplifier turn-on/off sequences are also supplied from the if group control area. The computer link between main control consoles to the group control area provides necessary functions (listed in Figure 6). For diagnostic purposes beam phase error, radial position error and synchronization phase errors are stored by the DSPs in a separate memory. Values are read by the main ring control computers. The multiple transfer sequence is supplied to the timing modules to generate delays ' $\mathrm{d}_{1}$ ', ' $\mathrm{d}_{2}$ ', .. etc.

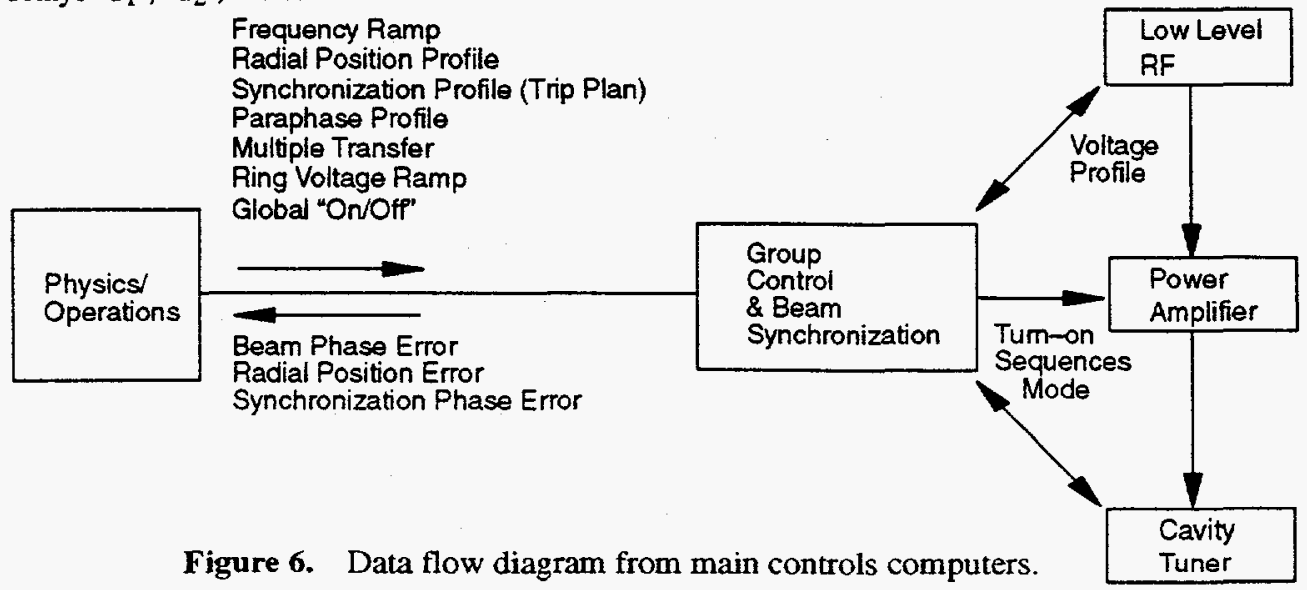




\section{SOME EXPERIMENTAL RESULTS}

Several parasitic beam tests were done at Fermilab to study the feasibility of the digital hardware. Also, we have demonstrated the phase-locking capabilities of the beam phase loop by accelerating the booster beam. We achieved an efficiency indistinguishable from that of the existing booster system. ${ }^{5}$ In addition to this in Figure 7 the bench test results of the synchronization loop are shown. The experiment was carried out with the hardware configuration discussed in Figure 6 of Reference 2 for phase locking two fixed frequencies ( $60 \mathrm{MHz}$ ). The $\mathrm{x}$-axis shows the phase error in degrees of the if wave after subtracting the phase between the DDS if signal and the fixed frequency with the trip-plan. The $y$-axis shows the number of samples. Clearly, on most occasions the loop was able to control the phase to within 3 degrees (equivalent to about $4 \mathrm{cms}$ wave length). A $20 \mathrm{ps}$ TDC was used for the time interval measurement.

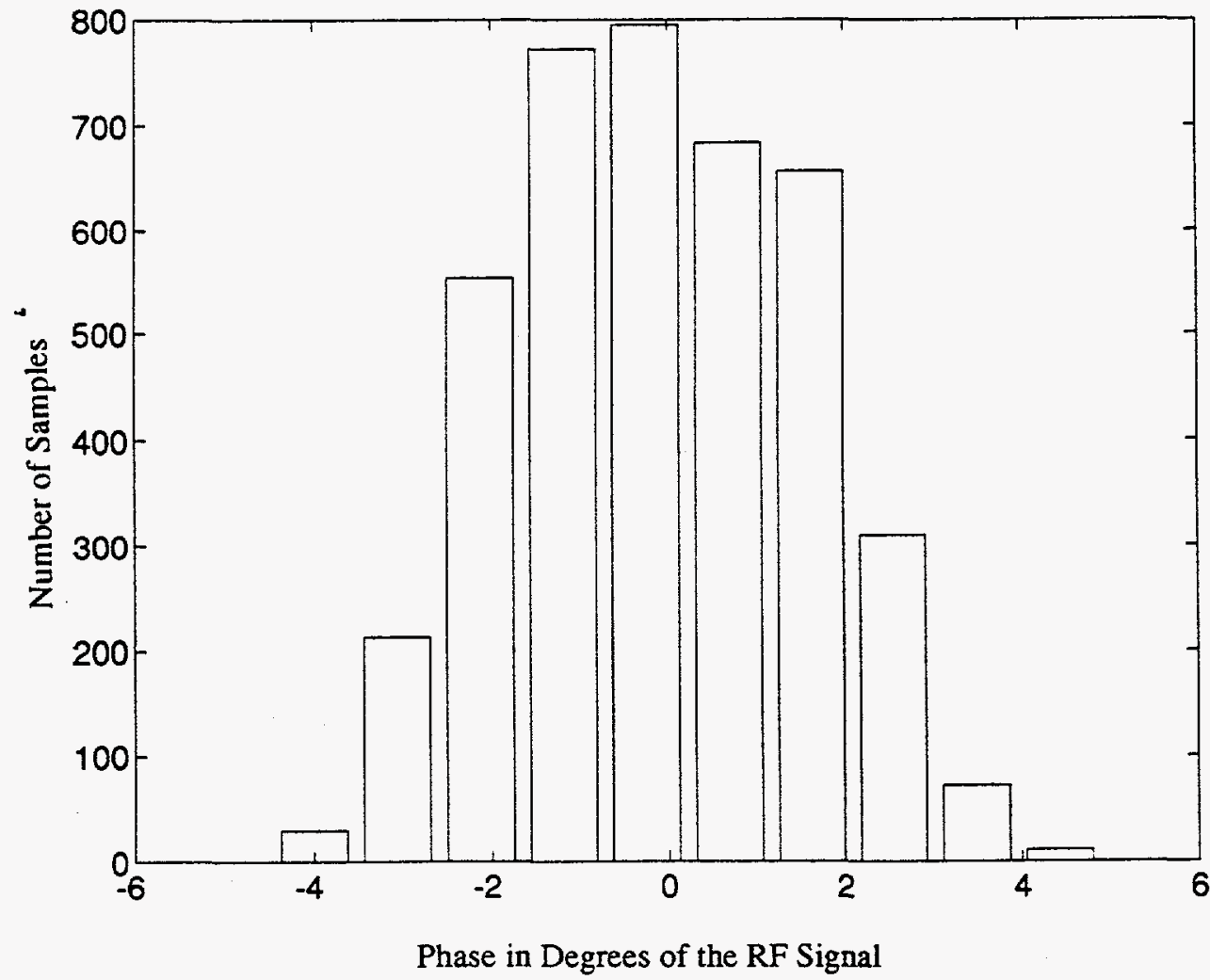

Figure 7. Histogram of the phase values between two RF frequencies when synchronized using trip-plan approach.

\section{CONCLUSIONS}

We believe the system proposed in Figure 1 can provide the precise reference phase and frequency needed for the LEB RF System which is necessary to accelerate the beam without beam loss, allow programmed beam radial position control, capture the linac beam adiabatically and guarantee synchronism with the MEB between a selected LEB bunch and a selected MEB bucket. We have also shown briefly how the beam control system can function with the precision timing system to provide synchronous triggers for extraction, how the if signals are distributed to each of the LEB RF stations and a pathway to interface with the controls computers.

\section{REFERENCES}

1. D.J. Martin et al., "Early Instrumentation Projects at the SSC," 2nd International Industrial Symposium on the Super Collider, Miami, March 14-16, 1990.

2. L.K. Mestha et al., "Early Stages in the Development of the Global RF Feedback for the SSC Low Energy Booster," 3rd International Industrial Symposium on the Super Collider, Atlanta, Georgia, March 13-15, 1991.

3. R.C. Webber, "Longitudinal Emittance, An Introduction to the Concept and Survey of Measurement Techniques Including Design of a Wall Current Monitor" AIP Conference Proceedings, 212, Accelerator Instrumentation, Upton, NY 1989.

4. G. Roberto et al., "Log-Ratio Technique for Beam Position Monitor Systems," EPAC, Berlin, 1991.

5. L.K. Mestha et al., "A Digital Beam Phase Loop for the Low Energy Booster," Proceedings of the IEEE Particle Accelerator Conference, Washington, D.C., May 17-20, 1993. 